\title{
Combining vision and touch in texture perception
}

\author{
BILL JONES and SANDRA O'NEIL \\ Carleton University, Ottawa, Ontario, Canada
}

\begin{abstract}
In two completely randomized experiments, subjects were required to judge either which was the rougher of two abrasive papers or whether two abrasive papers were the same or different. Judgments were made visually, tactually, or with both vision and touch available. The subjects used either the right hand or the left hand in the touch conditions. Differences between the hands in terms of either proportion correct or mean latency were negligible in both experiments. Accuracy was statistically equivalent across conditions, although the latency of visual judgments was shorter. In the same-different experiment, comparable accuracy for vision and touch appeared to result from different strategies. Subjects in the touch condition were much less likely to be correct without guessing on "different" trials. In a third, within-subject experiment, a comparison was made of four probability models of dual-mode efficiency. Subjects appeared not to treat the two sources of information as independent; rather, the probability of a correct response in the combined vision-touch condition could be best described as the arithmetic mean of the vision and touch conditions. Latencies for the combined condition also appeared to reflect a similar compromise. Implications for further research are discussed.
\end{abstract}

How do we use visual and tactile information when we make judgments about the characteristics of objects? One answer to this question is that since we rely primarily upon vision, vision comes to dominate touch. Dominance may have at least two distinct senses. Either we judge objects more efficiently (more accurately, more rapidly) when we see them than when we explore them with the hands (haptics; e.g., Jones, 1981) or, in a dual-mode condition, visual information is more heavily weighted (e.g., Jones, 1983; Warren \& Schmitt, 1978; Welch \& Warren, 1980), perhaps to the point that nonvisual information is virtually ignored (Rock \& Victor, 1964). While these two notions of dominance are conceptually independent, in practice it may be that one source of information is the more heavily weighted because it is ordinarily found to be more reliable or more precisely graded.

The evidence is clear that visual judgments of form are made more efficiently than the corresponding haptic judgments. Jones (1981) reviewed 50 matching-to-sample experiments and found that, in all but two, visual matching was more accurate than haptic matching. In the two exceptions, vision and touch were about as accurate. Jones (1981) also argued that the pattern of results in visualhaptic cross-modal matching experiments is predictable from the superiority of visual pickup.

There is also ample evidence that visual information is more heavily weighted than haptic information in a partial judgment (e.g., Jones, 1983; Welch \& Warren, 1980). Jones (1983) directly studied the applicability of a

This research was supported by a grant from NSERC to the senior author. The authors' mailing address is: Department of Psychology, Carleton University, Ottawa, Ontario, Canada K1S 5B6. weighted averaging model to rating-scale judgments of the width of two cubes when both were seen, both were felt, or one was felt and one was seen. The model held for both visual and haptic judgments, and the derived psychophysical function for length was linear in both cases. However, when subjects could both see and feel the stimuli, visual information received a somewhat higher weighting.

Taylor, Lederman, and Gibson (1973) have argued that form is essentially not suited to perception by touch; rather, touch is primarily suited to texture perception. It is possible, therefore, that visual dominance in either sense may not be observed for visual-tactual judgments of texture. Heller (1982) found that accuracy of discrimination of smoothness is about the same for vision and touch (if anything, subjects were less accurate in the visual conditions). Lederman and Abbott (1981) have shown that psychometric and psychophysical functions for visual, tactual, and combined visual-tactual scaling of roughness are very similar. They also found that when subjects were presented with discrepant information through vision and touch, judgments of surface roughness could be described as a compromise between the two sources. This is obviously quite different from the frequently cited demonstration by Rock and Victor (1964) showing that the length of an object viewed through a minifying lens at the same time as it is grasped is judged essentially according to its visual appearance. In short, there is evidence that when subjects perceive some aspects of surface texture, we do not observe visual dominance over touch, either in the sense that visual information is more heavily weighted or in the sense that visual judgments are more accurate. 
As always, there are some contradictory results. Björkman (1967) used the variability of same-different judgments as an index of efficiency in discriminating sandpapers. Visual judgments were markedly less variable than tactual judgments. Brown (1960) also found that subjects tended to pick the rougher of two wooden surfaces more accurately and more rapidly through visual inspection. However, performance in dual-mode conditions depended upon how the samples were illuminated. Lighting that eliminated the visual grain-shadow in the samples produced performance that was equivalent to purely tactual matching. Lighting that was appropriate for visual matching resulted in dual-mode performance that corresponded to the efficiency of purely visual matching. In other words, subjects appeared to use the better source of information in the dual-mode conditions rather than relying exclusively upon visual cues as they appear to do when spatial judgments are in question (Rock \& Victor, 1964).

Clearly more detailed examinations of visual (V), tactual (T), and visual-tactual (VT) judgments of surface texture are needed. The first two experiments here examine speed and accuracy to such judgments in a 2-AFC and in a same-different paradigm. A subsidiary question in the first two experiments reported here concerns possible differences between the hands in VT judgments. Although there is evidence from a number of different experimental paradigms (Lederman, Jones, \& Segalowitz, 1984 ) that right-handers make very similar judgments of surface roughness with either hand, there is some reason to think that VT judgments should differ according to which hand is used. Semmes (1968) has argued that the right hemisphere is more diffusely organized than the left in a way that would facilitate cross-modal connections. Given that information picked up by each hand is processed, in the main, by the contralateral hemisphere, VT judgments might be made more efficiently when the left hand is used.

\section{EXPERIMENT 1}

In this experiment, subjects were required to choose the rougher of two abrasive papers that differed in grit value. The grit value is proportional to the number of openings in a sieve used to sort the particles that make up the abrasive surface. The fewer the openings, the larger the particles. Lower grit values should therefore feel rougher. Judgments were made under three basic conditions $(\mathrm{V}, \mathrm{T}$, and VT). The actual information was obtained with either the right hand $\left(T_{R}\right.$ and $\left.V T_{R}\right)$ or the left hand $\left(\mathrm{T}_{\mathrm{L}}\right.$ and $\left.\mathrm{VT}_{\mathrm{L}}\right)$.

\section{Method}

Subjects. The subjects were 45 ( 21 male and 24 female) righthanded undergraduates who participated in the experiment for course credit. Handedness was assessed by means of a standard inventory (Oldfield, 1971).

Stimuli and Apparatus. The stimuli were $3-\mathrm{cm}^{2}$ squares of abrasive papers with grit values of $80,100,120,150,180,220,240$,
280,320 , and 400 . Since the different grit values as manufactured also differ in brightness, the samples were all sprayed with a matt black paint to eliminate brightness as a possible cue to texture. Two different grit samples were arranged in pairs on rectangular panels such that there were no more than three steps on the scale of grit values between the samples. The number of steps presumably determines to a degree the difficulty of the discrimination.

In the $\mathrm{T}$ conditions, the samples were hidden from view by means of a wooden baffle under which the subject placed one hand. The subjects wore a work glove on this hand, with the index and second fingers cut off to permit exploration. They also wore acoustic earmuffs to attenuate any auditory texture cues (see Lederman, 1979). The latency of the subjects' responses was timed by means of locally constructed equipment (clock and voice key). The room in which the experiment took place was illuminated by fluorescent strips in the ceiling.

Procedure. Subjects were assigned at random to one of five conditions $\left(V, T_{R}, T_{L}, V T_{R}\right.$, and $\left.V T_{L}\right)$ such that there were nine subjects per condition. On each trial, the subject was required to choose the "rougher" of the two samples by saying "This one" to stop the clock and simultaneously touching the appropriate sample. The clock was started either when the subject touched the sample in conditions involving touch or when the experimenter placed the sample in front of the subject. The stimuli were selected at random on each trial as were the number of steps, 1,2 , or 3 , between grit values. The position of the lower grit value on the right or left of the panel was also randomized from trial to trial. In each condition, the subjects performed a sequence of 132 trials.

\section{Results}

Differences between hand conditions were all nonsignificant, and the results are presented as averages across the hands. The mean proportion correct, $\mathrm{P}(\mathrm{C})$, is shown in Figure 1 as a function of the number of steps, 1, 2, or 3 , between grit values for V, T, and VT conditions. Analysis of variance indicated that the conditions did not differ significantly $[F(4,40)=1.18]$. Accuracy increased significantly as a function of the number of steps $[F(2,80)$

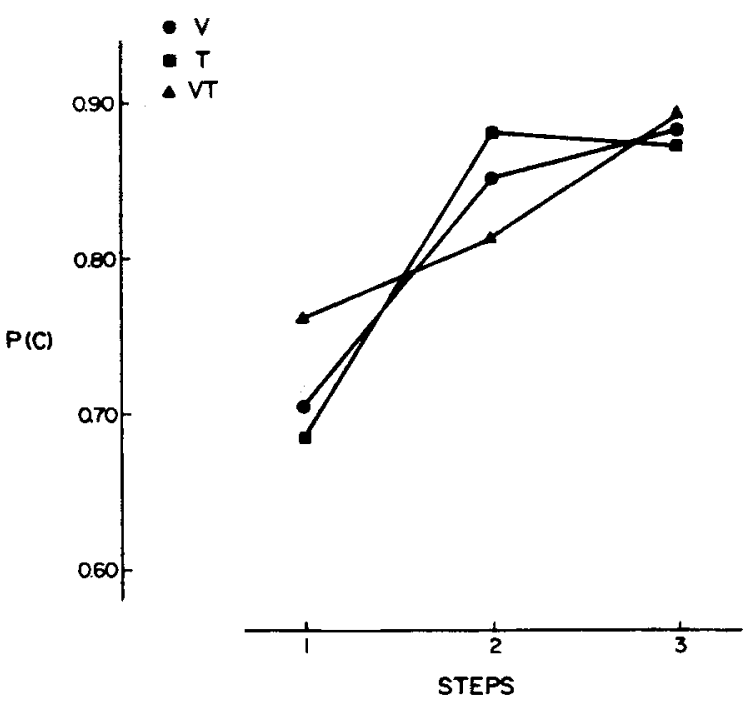

Figure 1. Proportion correct, $P(C)$, as a function of difficulty (steps), in Experiment 1. The solid lines represent the right-hand conditions $\left(T_{R}\right.$ and $\left.V T_{R}\right)$, and the dotted lines, the left-hand conditions $\left(T_{L}\right.$ and $\left.V T_{L}\right)$. 
Table 1

Response Latency Means and Standard Deviations (in Seconds) for V, T, and VT Matching When Subjects Were Correct, in Error, and Overall (Experiment 1, 2-AFC)

\begin{tabular}{lrrrrrrr} 
& \multicolumn{2}{c}{ Correct } & & \multicolumn{2}{c}{ Error } & \multicolumn{2}{c}{ All } \\
\cline { 2 - 5 } \cline { 6 - 8 } & Mean & SD & & Mean & SD & Mean & SD \\
\hline V & .95 & .45 & & 1.14 & .96 & .98 & .52 \\
T & 3.00 & 1.29 & & 4.18 & 2.14 & 3.21 & 1.40 \\
VT & 1.75 & .62 & & 2.30 & .91 & 1.85 & .66 \\
\hline
\end{tabular}

$=117.18, \mathrm{p}<.001]$, and the two factors interacted significantly $[F(8,80)=4.74, p<.01]$. The interaction is clear in Figure 1. Accuracy increases across the three steps for the two VT conditions and tends to level off at two steps for the $\mathrm{V}$ and $\mathrm{T}$ conditions. Accuracy averaged across steps was equivalent, with mean $\mathrm{P}(\mathrm{C})=.81$ for the five conditions.

Table 1 shows, however, that response times tended to be shorter for visual judgments. Correct response latencies were also shorter than the latencies for errors for each condition. This result appears to be typical of situations in which subjects trade speed for accuracy (e.g., Swensson, 1972). Since there were no interactions between conditions and steps, the data were collapsed across the factor of steps. Analysis of variance showed a significant difference between the five conditions $[F(4,40)=8.14$, $\mathrm{p}<.001]$. Newman-Keuls tests showed that mean latencies for the two $T$ conditions were significantly longer than all other conditions, with the exception that $\mathrm{VT}_{R}$ did not differ significantly from $T_{R}$.

Correct responses were made significantly faster than errors $[F(1,40)=38.50, p<.001]$, and there was a small, but significant, interaction between the factors $[F(4,40)=3.38, p<.03]$. This interaction appears to result from a somewhat greater difference between correct response and error latencies in the $T$ conditions. Newman-Keuls tests indicated a significant difference between correct response and error latencies only for tactual matching.

In sum, paired comparisons of surface roughness were made about as accurately by the subjects who viewed the stimuli as by those who felt the stimuli or could make use of dual-mode information. However, the subjects required from 2 to 3 times longer in the $T$ conditions to achieve the level of accuracy achieved in the $\mathrm{V}$ condition. The fact that correct responses were made significantly faster than errors in both $T$ conditions suggests similarly that subjects were more likely to trade speed to obtain accuracy when texture information was obtained through touch (cf. Swensson, 1972). Finally, there was no sign of a difference between right- and left-hand involvement in the VT condition for the present procedure.

\section{EXPERIMENT 2}

The second experiment was similar to the first, except that the pairs of grit values were either the same or different, unlike the previous experiment, in which all of the stimuli were different. This procedure allows a somewhat more fine-grain analysis.

\section{Method}

The subjects were 45 right-handed undergraduates ( 22 males and 23 females) who had not participated in Experiment 1. Handedness was again assessed using a questionnaire (Oldfield, 1971). Stimulus pairs were prepared such that the two grit values were either the same (all grit values were used) or different, as before. On any trial, "same" or "different" values were selected at random and the particular pair of stimuli was also chosen at random. The subject responded "same" or "different." Otherwise, the procedure was identical to that of Experiment 1.

\section{Results}

Again, no effect of hand was found and the data presented are averaged across hand condition. Mean $P(C)$ values for "same" and "different" trials are shown in Table 2. Analysis of variance indicated no significant difference between $V, T$, or VT conditions $[F(4,40)=$ 1.91]. The subjects were significantly more likely to be correct on "same" trials $[\mathrm{F}(1,40)=12.32, \mathrm{p}<.001]$, although there was a significant interaction between conditions and the type of trial $[F(4,40)=5.46, p<.001]$. The table shows how the interaction arises. Proportions correct for the two types of trials were almost equal in the $\mathrm{V}$ condition and at least $13 \%$ different in the other conditions.

This suggests a strategic difference between vision and the conditions involving touch. A correct response independent of guessing on a "different" trial requires precise discernment of the features that distinguish the two samples. Subjects may be better able, or perhaps simply more willing, to search for differentiating features in the purely visual condition. This suggestion will be explored below.

Latency data are shown in Table 3. Visual response tended to be faster than responses in the other conditions. With the exception of one comparison, "different" trials in the $T_{L}$ condition, correct responses were again consistently faster than errors. Responses on "same" trials tended to be somewhat slower across the board than they were on "different" trials. Analysis of variance showed a small effect of conditions $[F(4,40)=2.26, p<.08]$. Responses on "same" trials were significantly slower, by about $25 \mathrm{msec}$, than on "different" trials $[F(1,40)=$ $14.22, \mathrm{p}<.001]$, and correct responses were significantly faster than errors, by about the same amount $[\mathrm{F}(1,40)=7.51, \mathrm{p}<.01]$.

So far, we have provided separate analyses of response

Table 2

Means and Standard Deviations for $P(C)$, for Same and Different Trials, and Overall for V, T, and VT Judgments (Experiment 2)

\begin{tabular}{|c|c|c|c|c|c|c|}
\hline & \multicolumn{2}{|c|}{ Same } & \multicolumn{2}{|c|}{ Different } & \multicolumn{2}{|c|}{ All } \\
\hline & Mean & SD & Mean & SD & Mean & SD \\
\hline V & .72 & .20 & .75 & .10 & .74 & .07 \\
\hline$T$ & .81 & .13 & .53 & .13 & .67 & .08 \\
\hline VT & .81 & .12 & .67 & .13 & .73 & .05 \\
\hline
\end{tabular}


Table 3

Response Latency Means and Standard Deviations (in Seconds) for V, T, and VT Matching, Same and Different Trials, Correct Responses and Errors, and Overall

\begin{tabular}{|c|c|c|c|c|c|c|c|c|c|c|}
\hline & \multicolumn{4}{|c|}{ Same } & \multicolumn{4}{|c|}{ Different } & \multirow{2}{*}{\multicolumn{2}{|c|}{ All }} \\
\hline & \multicolumn{2}{|c|}{ Correct } & \multicolumn{2}{|c|}{ Error } & \multicolumn{2}{|c|}{ Correct } & \multicolumn{2}{|c|}{ Error } & & \\
\hline & $\overrightarrow{\text { Mean }}$ & $\overline{\mathrm{SD}}$ & Mean & SD & Mean & SD & Mean & SD & Mean & $\mathrm{SD}$ \\
\hline V & 1.21 & .66 & 1.49 & 1.25 & 1.04 & .59 & 1.13 & .63 & 1.14 & .68 \\
\hline $\mathrm{T}$ & 2.89 & 1.82 & 3.20 & 1.19 & 2.86 & 1.62 & 2.85 & 1.54 & 2.82 & 1.86 \\
\hline VT & 2.40 & 1.65 & 2.74 & 1.83 & 2.09 & 1.28 & 2.48 & 1.68 & 2.29 & 1.48 \\
\hline
\end{tabular}

Table 4

Estimates of $\mathbf{p}$ and $\mu$, Response Probabilities, and Latencies (in Seconds) Corrected for Guessing (Link, 1982) for Same and Different Trials (Experiment 2)

\begin{tabular}{llllll}
\hline & \multicolumn{2}{c}{ Same } & & \multicolumn{2}{c}{ Different } \\
\cline { 2 - 3 } \cline { 5 - 6 } & $\mathrm{p}$ & $\mu$ & & $\mathrm{p}$ & $\mu$ \\
\hline $\mathrm{V}$ & .44 & 1.03 & & .50 & 1.00 \\
$\mathrm{~T}$ & .62 & 2.80 & .08 & 2.92 \\
$\mathrm{VT}$ & .62 & 2.30 & .34 & 1.71 \\
\hline
\end{tabular}

probabilities and latencies. In other words, we have assumed that the two statistics are independent, an assumption which Link (1982), among others (e.g., Swensson, 1972), has shown is simplistic at best. Link argues for a two-state model of binary classification of stimuli into, for example, same and different. Either the subject enters, with probability $\mathrm{p}$, a rate that leads with certainty to a correct response or the subject enters a second mutually exclusive state, with associated probability $1-p$, in which he or she may guess correctly or incorrectly. The problem now is to estimate the value of $p$ of $\mu$, the mean time taken to make a correct response without guessing. Link provides estimates of $\mathrm{p}$ and $\mu$ under some simple assumptions. ${ }^{1}$

The two statistics were computed separately for "same" and "different" trials, and the values are shown in Table 4

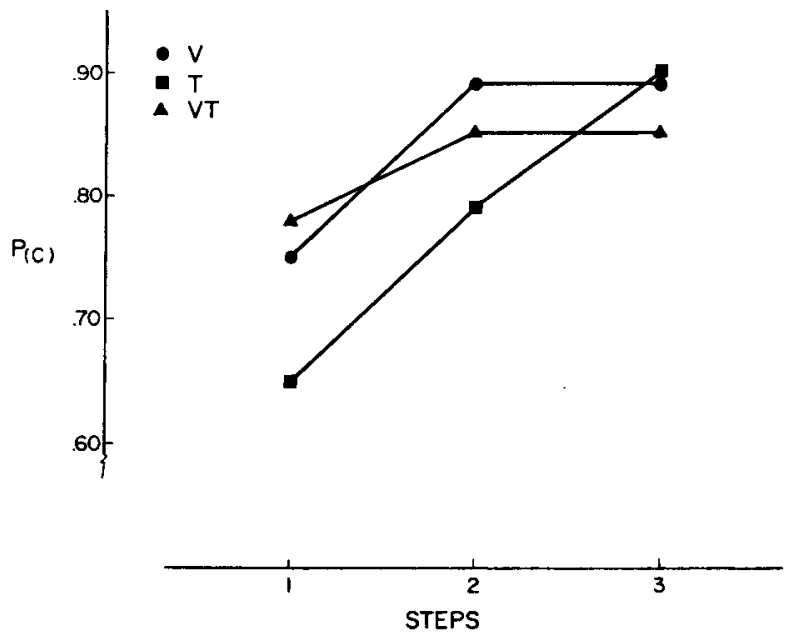

Figure 2. Proportion correct, $P(C)$, as a function of difficulty (steps) in Experiment 2. collapsed across the hands in the T and VT conditions. The principal features of this analysis are that response probabilities and latencies for the two types of trial are quite similar for the visual condition (if anything, subjects were more likely to guess on "same" trials), whereas in tactual conditions subjects were rarely correct without guessing on "different" trials. The VT conditions represent a compromise. Response probabilities and latencies are intermediate between the $\mathrm{V}$ and $\mathrm{T}$ values.

To summarize, the results of this experiment were comparable to the results of Experiment 1. Visual judgments were no more accurate than tactual judgments on those made in the dual-mode condition, although they tended to be somewhat faster, particualrly when response probabilities and latencies were corrected for guessing. When information was obtained through touch, the subjects appeared much less likely to discern differentiating features on "different" trials. However, there was no indication that vision dominated touch. As in Experiment 1, there were again no effects due to the hand used to obtain haptic information.

\section{EXPERIMENT 3}

This experiment is a direct examination of some simple probability models of VT judgments which have been found useful in other studies of intersensory processing (e.g., Craig, Colquhoun, \& Corcoran, 1976).

Heller (1982) has speculated that superior VT accuracy in his experiment was due to the fact that vision and touch normally cooperate, and that, under purely visual or purely tactual conditions, subjects will presumably be deprived of information they would ordinarily use to make judgments about surface features. Unfortunately, dualmode performance superior to either single mode condition does not by itself demonstrate'that vision and touch in some sense cooperate. In fact, greater dual-mode accuracy will arise if vision and touch operate independently in the dual-mode condition. Assuming this to be the case, and letting $P$ be the probability of a correct response, the probability correct in the VT condition, $\mathrm{P}_{\mathrm{VT}}$, is given by the "statistical summation"' of $P_{V}$ and $P_{T}$, the probabilities correct for vision and touch, respectively:

$$
\mathbf{P}_{\mathrm{VT}}=\mathbf{P}_{\mathbf{V}}+\mathbf{P}_{\mathbf{T}}-\mathbf{P}_{\mathrm{V}} \mathbf{P}_{\mathrm{T}},
$$

which implies that $P_{v t}$ is greater than or equal to $P_{v}$ or $\mathrm{P}_{\mathrm{T}}$. The evidence available from vision and hearing is that an equation analogous to Equation 1 considerably overpredicts dual-mode frequency (e.g., Craig et al., 1976; Loveless, Brebner, \& Hamilton, 1970). Equation 1 will be referred to as the independence model.

Complete dominance, in the sense that information from the less reliable or less finely graded system is simply ignored, may be modeled by

$$
P_{v T}=\max P,
$$


where $\max P$ is the greater of $P_{V}$ or $P_{T}$. This equation, which will be referred to as the dominance model, is suggested by discrepancy studies of spatial judgments (e.g., Rock \& Victor, 1964). In the case of texture perception, Lederman and Abbott's (1981) discrepancy experiment and the data from Experiments 1 and 2 imply that $P_{v T}$ reflects a compromise between the systems. Perhaps the simplest compromise is that $P_{V T}$ is the arithmetic mean of $\mathrm{P}_{\mathrm{V}}$ and $\mathrm{P}_{\mathrm{T}}$,

$$
P_{v T}=\left(P_{V}+P_{T}\right) / 2 .
$$

Formally, both Equations 2 and 3 are special cases of a general weighted averaging model in which $P_{V}$ and $P_{T}$ are associated with weight parameters such that the sum of the weights is unity. Equation 2 is generated by setting the weight parameter associated with the lesser of $\mathrm{P}_{\mathrm{V}}$ or $\mathrm{P}_{\mathrm{T}}$ to zero; Equation 3 is generated by setting both values equal to one-half. Nonetheless, empirical implications of the two equations are quite distinct and it is worth considering them separately.

Finally, it is worth considering an equation that has provided a good fit in the case of combined visual-auditory detection and recognition (Craig et al., 1976) and in other contexts (Jones, 1982). If we assume that visual and haptic texture information is correlated, we modify Equation 1 as follows

$$
\mathrm{P}_{\mathrm{VT}}=\mathrm{P}_{\mathrm{V}}+\mathrm{P}_{\mathrm{T}}-\mathrm{P}_{\mathrm{V}} \mathrm{P}_{\mathrm{T}}-\operatorname{cov} \mathrm{VT},
$$

where cov VT $=\phi\left[\mathrm{P}_{\mathrm{V}}\left(1-\mathrm{P}_{\mathrm{V}}\right) \mathrm{P}_{\mathrm{T}}\left(1-\mathrm{P}_{\mathrm{T}}\right)\right]^{1 / 2}$ and $\phi$ is a correlation coefficient such that $0 \leq \phi \leq 1$ and $E(\phi)$ $=.5$ (Craig et al., 1976). We shall refer to Equation 4 as the covariance model.

In the following experiment, subjects using the right hand for tactual pickup were tested in three conditions, $\mathrm{V}, \mathrm{T}$, and VT, to allow predictions of $\mathrm{P}_{\mathrm{VT}}$ under Equations $1,2,3$, and 4 to be generated for each subject.

\section{Method}

The subjects were 18 ( 9 male, 9 female) right-handed undergraduates, who again participated for course credit. The procedure of Experiment 1 (2-AFC) was followed, except that the design was within-subject and the conditions $T_{L}$ and $V_{T} T_{L}$ were not run. Three subjects were assigned to each of the six possible orders of the three conditions, $\mathrm{V}, \mathrm{T}$, and VT.

\section{Results}

Proportions correct for each condition as a function of the number of steps on the scale of grit values are shown in Table 5. It is apparent that the results are comparable to those of Experiment 1. Accuracy in the T conditions improved across the three levels of difficulty; asymptotic accuracy for the other two conditions was achieved by step 2. Analysis of variance showed significant main effects of conditions $[F(2,34)=5.81, \mathrm{p}<.01]$ and levels of difficulty $[F(2,34)=48.45, p<.001]$, and a signifi-
Table 5

Mean Proportion Correct in Experiment 3 for V, T, and VT Conditions from the Three Levels of Difficulty and

\begin{tabular}{|c|c|c|c|c|c|c|c|c|}
\hline & \multicolumn{6}{|c|}{ Level } & \multirow{2}{*}{\multicolumn{2}{|c|}{ All }} \\
\hline & \multicolumn{2}{|c|}{1} & \multicolumn{2}{|c|}{2} & \multicolumn{2}{|c|}{3} & & \\
\hline & Mean & SD & Mean & SD & Mean & SD & Mean & SD \\
\hline V & .75 & .09 & .89 & .08 & .89 & .08 & .84 & .06 \\
\hline $\mathbf{T}$ & .66 & .09 & .79 & .09 & .90 & .07 & .77 & .05 \\
\hline VT & .76 & .10 & .85 & .10 & .85 & .09 & .82 & .05 \\
\hline
\end{tabular}
Average Across Difficulty

cant interaction $[F(4,68)=6.20, p<.001]$. NewmanKeuls showed significant differences between the three levels and that the $T$ condition was significantly less accurate than V and VT.

Predicted values of $P_{V}$ and $P_{T}$ were obtained for each subject at each difficulty level. The sum of the squared differences, $\Sigma \mathrm{d}^{2}$, between observed and predicted values across the 18 subjects then constitutes a measure of the goodness of fit of each equation. The relative goodness of fit is the sum for any equation divided by the minimum sum obtained. The variance of the squared differences was also obtained together with the relative variance. These values are shown in Table 5. Overall, the assumption that visual and haptic texture information are treated independently clearly provided the worst fit. Equation 1 overpredicted $P_{V t}$ in the case of every subject. The assumptions either that one source of information is ignored (Equation 2) or that the two sources covary (Equation 4) provided fits that were about as good. The arithmetic mean of $\mathbf{P}_{\mathbf{v}}$ and $\mathbf{P}_{\mathrm{T}}$ is, however, the best fitting equation and also shows the smallest variance.

Conceivably, visual and tactual roughness information may be combined in different ways according to the difficulty of the comparison. However, Table 6 also shows the goodness of fit and variance statistics for each model at each level of difficulty. The arithmetic mean of $\mathrm{P}_{\mathrm{V}}$ and $\mathrm{P}_{\mathrm{T}}$ provides the best fit for each level and, with the exception of level 1, Equation 3 also resulted in the least variable set of predictions.

In short, we can reasonably reject the assumption that subjects treat visual and haptic texture information as in dependent. Rather, accuracy of discrimination of roughness when both vision and touch are available may be best considered as resulting from an averaging process.

Figure 2, which depicts mean latencies for each condition as a function of level of difficulty, appears to show that mean latencies in the VT condition ( VVT $_{\text {) }}$ can also be reasonably regarded as a compromise between the mean latencies for the $\mathrm{V}$ and $T$ conditions ( $\mathrm{L}_{V}$ and $\mathrm{L}_{T}$, respectively), since $L_{V T}$ is intermediate between $L_{V}$ and $\mathbf{L}_{\mathrm{T}}$ for all three levels. Analysis of variance showed significant main effects of conditions $[F(2,34)=14.47$, $\mathrm{p}<.001]$ and level $[\mathrm{F}(2,34)=21.01, \mathrm{p}<.001]$, but no significant interaction. The three conditions all differed significantly from each other, as did the three levels of difficulty (Newman-Keuls). 
Table 6

Indices of Goodness of Fit $\left(\Sigma d^{2}\right)$ and Variability (Var $\left.d^{2}\right)$ for Each Model for Each Level of Difficulty and Overall

\begin{tabular}{|c|c|c|c|c|}
\hline & $\begin{array}{c}\text { Equation 1 } \\
\text { Independence }\end{array}$ & $\begin{array}{l}\text { Equation } 2 \\
\text { Dominance }\end{array}$ & $\begin{array}{c}\text { Equation } 3 \\
\text { Mean }\end{array}$ & $\begin{array}{l}\text { Equation } 4 \\
\text { Covariance }\end{array}$ \\
\hline \multicolumn{5}{|c|}{ Level 1} \\
\hline$\Sigma \mathrm{d}^{2}$ & .5834 & .2373 & .2002 & .2142 \\
\hline Relative & 2.91 & 1.14 & 1 & 1.07 \\
\hline $\operatorname{Var} d^{2}$ & .0012 & .00013 & .00018 & .00024 \\
\hline Relative & 9.23 & 1 & 1.38 & 1.81 \\
\hline \multicolumn{5}{|c|}{ Level 2} \\
\hline$\Sigma d^{2}$ & .3416 & .3082 & .2433 & .3072 \\
\hline Relative & 1.40 & 1.27 & 1 & 1.26 \\
\hline $\operatorname{Var} d^{2}$ & .0006 & .00041 & .00017 & .00153 \\
\hline Relative & 3.53 & 2.14 & 1 & 3.12 \\
\hline \multicolumn{5}{|c|}{ Level 3} \\
\hline$\Sigma \mathrm{d}^{2}$ & .5237 & .3811 & .3276 & .4218 \\
\hline Relative & 1.60 & 1.19 & 1 & 1.29 \\
\hline $\operatorname{Var} d^{2}$ & .00100 & .00094 & .00070 & .00112 \\
\hline Relative & 1.43 & 1.34 & 1 & 1.60 \\
\hline \multicolumn{5}{|c|}{ Overall } \\
\hline$\Sigma \mathrm{d}^{2}$ & .4240 & .1661 & .0683 & .1476 \\
\hline Relative & 6.12 & 2.43 & 1 & 2.16 \\
\hline $\operatorname{Var} d^{2}$ & .00028 & .00033 & .00002 & .00012 \\
\hline Relative & 14 & 16.50 & 1 & 5.97 \\
\hline
\end{tabular}

There was no evidence at any level for any subject of facilitation of $L_{V T}$ in the sense that $L_{V T}$ would be faster than the minimum of $L_{V}$ and $L_{T}$ (see, e.g., Nickerson, 1973). Such facilitation does occur in visual-auditory detection and recognition experiments (e.g., Craig et al., 1976; Nickerson, 1973). In these cases, visual and auditory response latencies tend to be very similar, and it can be assured either that peripheral processes require a comparable amount of time in the two cases or at least that variation in the latencies is affected to only a minimal degree by variation in the peripheral processes. When we compare vision and touch, however, neither assumption is plausible. Mean latencies for vision and touch in the present experiment differed by a factor of two. In the nature of things, it will take longer to run one's fingers over an object than it will to scan the same object visually.

\section{GENERAL DISCUSSION}

In general, we found that visual and haptic judgments of surface roughness were made with comparable accuracy, although visual judgments, since they were effected more rapidly, could be described as the more efficient. This confirms the result observed by Brown (1960), using wood surfaces.

Unlike experiments that have compared visual and auditory detection or recognition with a dual-mode condition (e.g., Craig et al., 1976; Loveless et al., 1970), the dual-mode condition in these experiments was not superior to either single-mode condition, whether we compared proportions correct or latencies. Our results provide direct support for the contention of Lederman and Abbott (1981) that subjects given both visual and tactual information will judge roughness in a way that reflects a compromise between vision and touch. Experiment 3 provided an examination, in a more quantitative way, of this compromise. As we have noted, the notion "that vision and touch normally work in a cooperative manner" (Heller, 1982 , p. 340) is not necessarily supported by the result $P_{V T}>\max P$, since this might also arise from the complete independence of the two channels (Equation 1). In practice, however, we were able to reject the assumption of the independence of vision and touch in texture perception as well as the assumption that one channel was completely dominant (Equation 2). Vision and touch do appear to cooperate in the sense that $P_{V T}$ may be best regarded as the mean of $P_{V}$ and $P_{T}$. An analogous result also held, approximately, for the latencies in the three conditions.

Heller's (1982) data on vision and touch in texture perception are comparable to our visual-auditory dualmode results, since he did find that $P_{V T}$ was greater than either $\mathrm{P}_{\mathrm{V}}$ or $\mathrm{P}_{\mathrm{T}}$. In fact, Equation 1 would provide the best fit of the four models studied here to the mean data observed in Heller's Experiments 1 and 2. There are some differences between his procedures and ours that may be responsible for the differences in the two sets of results. Heller required subjects to choose the smoothest of three samples of abrasive paper. This 3-AFC procedure may have been more difficult than the 2-AFC procedure we used in Experiments 1 and 3, although overall accuracy in our experiments and Heller's appear roughly comparable. Perhaps asking subjects about the smoothness, as opposed to the roughness, of the samples may focus a subject's attention on different dimensions of the stimuli. Eisler and Edberg (1982) have shown that the visual perceptions of texture can be conveniently regarded as having a number of dimensions, including strength and order. The strength dimension orders clarity and unclarity, evenness and coarseness, small elements and large elements. The order dimension is associated with the regularity or irregularity of elements of the stimuli. Conceivably, requiring subjects to judge the roughness of surfaces may direct their attention predominantly to one dimension (strength?), whereas requiring judgments of smoothness directs attention to another dimension (order?). Further research is obviously necessary to determine how different instructions may affect strategies for judging texture and to show how efficiency of judgment in the dual-mode VT condition is associated with particular strategies.

Further research might also usefully focus on Equation 4, the weighted averaging model, of which Equation 3, the arithmetic mean model, is one special case. Weighted averaging models are, of course, familiar in perception from the work of Anderson (1974). A direct test of such a model could be made by requiring subjects to make rating-scale judgments of factorial combinations of roughness samples, one presented visually and one tactually (cf. Jones, 1983). 


\section{REFERENCES}

Anderson, N. H. (1974). Algebraic models in perception. In E. C. Carterette \& M. P. Friedman (Eds.), Handbook of perception (Vol. 2). New York: Academic Press.

BJöRKMAN, M. (1967). Relations between intra-modal and cross-modal matching. Scandinavian Journal of Psychology, 8, 67-76.

BRowN, I. D. (1960). Visual and tactual judgments of surface roughness. Ergonomics, 3, 51-61.

Craig, A., Colquhoun, W. P., \& Corcoran, D. W. J. (1976). Combining evidence presented simultaneously to the eye and the ear: A comparison of some predictive models. Perception \& Psychophysics, 19, 473-484.

Eisler, H., \& EdBerg, G. (1982). The visual perception of feature: A psychophysical investigation of an architectural problem. In B. Wegener (Ed.), Social attitudes and psychological measurement. Hillsdale, NJ: Erlbaum.

HeLler, M. A. (1982). Visual and tactual texture cooperation: Intersensory cooperation. Perception \& Psychophysics, 31, 339-344.

JONES, B. (1981). The developmental significance of cross-modal matching. In R. D. Walk \& H. L. Pick, Jr., Intersensory perception and sensory integration. New York: Plenum Press.

JONES, B. (1982). The integrative action of the cerebral hemispheres. Perception \& Psychophysics, 32, 423-453.

JONES, B. (1983). Psychological analyses of haptic and haptic-visual judgments of extent. Quarterly Journal of Experimental Psychology: Human Experimental Psychology, 35, 597-606.

Lederman, S. J. (1979). Auditory texture perception. Perception, 8, 93-103.

Lederman, S. J., \& ABbotT, S. G. (1981). Texture perception: Studies of intersensory organization using a discrepancy paradigm. Journal of Experimental Psychology: Human Perception and Performance, 7, 902-915.

Lederman, S. J., Jones, B., \& Segalowitz (1984). Lateral symmetry in the tactual perception of roughness. Canadian Journal of Psychology, 38, 599-609.

LiNK, S. W. (1982). Correcting response measures for guessing and partial information. Psychological Bulletin, 92, 469-486.
Loveless, N. E., Brebner, J., \& Hamilton, P. (1970). Bisensory presentation of information. Psychological Bulletin, 73, 161-199.

LUCE, R. D. (1963). Detection and recognition. In R. D. Luce, R. R. Bush, \& E. Galanter (Eds.), Handbook of mathematical psychology (Vol. 1). New York: Wiley.

NICKERSON, R. S. (1978). Intersensory facilitation of reaction time: Energy summation on preparation enhancement. Psychological Review, 80, 489-509.

OldFIELD, R. C. (1971). The assessment and analysis of handedness: The Edinburgh Inventory. Neuropsychologia, 9, 97-114.

RocK, I., \& VICTOR, J. (1964). Vision and touch: An experimentally created conflict between the two senses. Science, 143, 594-596.

Semmes, J. (1968). Hemispheric specialization: A possible clue to mechanism. Neuropsychologia, 6, 511-526.

Swensson, R. G. (1972). The elusive tradeoff: Speed vs accuracy in visual discrimination tasks. Perception \& Psychophysics, 12, 16-32.

TAYLoR, M. M., Lederman, S. J., \& Gibson, R. H. (1973). Tactual perception of texture. In E. C. Carterette \& M. P. Friedman (Eds.), Handbook of perception: (Vol. 3). Biology of perceptual systems. New York: Academic Press.

WARREN, D. H., \& SCHMiTT, J. L. (1978). On the plasticity of visualproprioceptive bias effects. Journal of Experimental Psychology: Human Perception and Performance, 4, 302-310.

WELCH, R. B., \& WARREN, D. H. (1980). Immediate perceptual response to intersensory discrepancy. Psychological Bulletin, 88, 638-667.

\section{NOTE}

1. Link's (1982) calculations are based upon a "high-threshold" model which may not hold in a wide variety of conditions. However, unbiased measures of accuracy calculated from Luce's (1963) choice theory show precisely the same pattern of results as discussed for $p$ here.

(Manuscript received June 1, 1984; revision accepted for publication December 18, 1984.) 